\title{
Edema agudo pulmonar posextubación
}

Cristhian Camilo Bastidas MD

Yamel Fuentes MD

Jacob García-Regalado MD'

\section{Post-extubation acute pulmonary edema}

\begin{abstract}
${ }^{a}$ Anestesiología y Reanimación Hospital Gr. Dr. Dario Fernández Fierro, Universidad Nacional Autónoma de México UNAM, Ciudad de México, México DF.

${ }^{b}$ Anestesiología y Medicina Critica, Universidad Nacional Autónoma de México UNAM., Hospital Dr. Darío Fernández Fierro. Ciudad de México, México. DF.

'Medicina Interna Universidad Nacional Autónoma de México UNAM, Unidad de Terapia Intensiva Hospital Regional Lic. Adolfo López Mateos, Ciudad de México, México DF.
\end{abstract}

\section{R ES U M E N}

El edema pulmonar agudo posextubación constituye un evento poco frecuente en la práctica anestésica. Cuando ocurre es necesario administrar tratamiento adecuado, ya que las complicaciones del mismo pueden traducirse en mayor estancia hospitalaria y lesiones serias del aparato respiratorio, como edema pulmonar intersticial, elevación de la presión hidrostática capilar y aumento de la presión transmural de las cámaras cardiacas, lo que puede conducir a fallo del aparato cardiovascular y a la muerte subsecuente del paciente. Se presenta el caso de un paciente con clasificación del estado físico ASA (American Society of Anesthesiologist) II, sin patología pulmonar previa, quien al momento de la emersión anestésica presenta un episodio agudo de dificultad respiratoria, con evidencia de edema pulmonar posextubación confirmado por clínica e imágenes con ulterior resolución satisfactoria.

Palabras clave: edema pulmonar agudo, posextubación, obstrucción de la vía aérea, presión negativa.

(C) 2021 Fundación Universitaria de Ciencias de la Salud - FUCS Este es un artículo Open Access bajo la licencia CC BY-NC-ND (http://creativecommons.org/licenses/by-nc-nd/4.0/).

\section{INFORMACIÓN DEL ARTÍCULO}

Historia del artículo: Fecha recibido: julio 22 de 2020 Fecha aceptado: abril 28 de 2021
Autor para correspondencia. Dr. Cristhian Camilo Bastidas cristhian.bastidasb@hotmail.com
DOI

10.31260/RepertMedCir.01217372.1024 
Post-extubation acute pulmonary edema is a rare event in anesthetic practice. When it occurs, it requires adequate treatment, since its complications may result in longer hospital stay and serious respiratory system lesions, such as interstitial pulmonary edema, increased capillary hydrostatic pressure and increased transmural pressure of the cardiac chambers, which may progress to cardiopulmonary arrest and death of the patient. We present the case of a patient who was classified with the (American Society of Anesthesiologist (ASA) II physical status, without a history of pulmonary disease, who upon emergence of general anesthesia developed an acute episode of respiratory distress, with evidence of post-extubation pulmonary edema confirmed by clinical and imaging findings with subsequent satisfactory resolution.

Key words: acute pulmonary edema, post-extubation, airway obstruction, negative pressure.

(C) 2021 Fundación Universitaria de Ciencias de la Salud - FUCS. This is an open access article under the CC BY-NC-ND license (http://creativecommons.org/licenses/by-nc-nd/4.0/).

IN T RODUCCIÓN

El edema pulmonar agudo de etiología no cardiogénica se desarrolla dentro de varias circunstancias y escenarios clínicos. Constituye un evento poco frecuente en la práctica anestésica que se reporta en algunos trabajos hasta de $0.1 \%{ }^{1}$ $\mathrm{Su}$ fisiopatogenia involucra una alteración a nivel de la membrana alveolocapilar, así como los factores que modulan el equilibrio de fluidos transcapilares. ${ }^{2,3}$ Su evolución puede comprometer de manera grave la integridad y funcionalidad respiratoria e incluso cuando no se aborda de manera oportuna puede llevar a fallo ventilatorio, hipoxemia severa y muerte. A nivel mundial es limitada la información epidemiológica acerca de la incidencia del edema pulmonar agudo posextubación asociado con anestesia general; varía ampliamente de acuerdo con las fuentes consultadas. Por lo anterior, no se cuenta con un protocolo establecido para minimizar su aparición.

La obstrucción de la vía aérea superior puede causarse por distintas etiologías, por ejemplo cuerpos extraños o laringitis y espasmo laríngeo en el ámbito de la anestesia y posextubación, lo cual desencadena una cadena de eventos que llevan a la presentación de un cuadro respiratorio agudo, cuyos datos hacen pensar en edema pulmonar no cardiogénico con un grado de severidad variable desde la autorresolución hasta la muerte. ${ }^{3}$

Se presenta un caso de edema agudo de pulmón no cardiogénico posextubación secundario a laringoespasmo que desarrolló hipoxemia grave. Se analizan los posibles factores de riesgo, la fisiopatología relacionada y el tratamiento. El objetivo principal de este reporte es describir la presentación del edema pulmonar agudo posextubación con su correspondiente manejo y evolución contrastado con la literatura disponible a nivel mundial.

\section{REPORTE DE CASO}

Paciente masculino de 36 años sin antecedentes de enfermedades crónicas degenerativas, con tabaquismo activo, (índice tabáquico 0.35) y tóxico exposicionales positivos de tipo laboral a contaminantes químicos $\mathrm{y}$ biomasa; paciente de profesión bombero.

Presenta cuadro clínico de 24 horas de evolución de dolor abdominal valorado por cirugía general con diagnóstico de apendicitis aguda. Al ingreso se ordenaron laboratorios, química sanguínea dentro de parámetros de normalidad, función renal conservada, ionograma sin alteración, biometría hemática con leucocitosis 15.600 y neutrofilia de $81.8 \%$, Hb $16 \mathrm{~g} / \mathrm{dL}$ Hto 49\%, plaquetas $335.000 \mathrm{U} /$ $\mathrm{dL}$, radiografía de tórax sin anormalidades (figura 1) y electrocardiograma con taquicardia sinusal sin otros agregados (figura 2).

Valorado por anestesiología se otorga clasificación de estado físico ASA II, con un riesgo respiratorio tipo II (complicaciones respiratorias 30\%) según escala de Castorena y riesgo cardiaco por escala de Goldman clase I (mortalidad cardiaca $0.2 \%$ ). La inducción anestésica se realiza con midazolam $2 \mathrm{mg}$ intravenoso, fentanil $200 \mathrm{mcg}$ IV, lidocaína $70 \mathrm{mg} I V$, propofol $140 \mathrm{mg} \mathrm{IV}$, cisatracurio $10 \mathrm{mg}$, sin complicaciones, se mantiene con sevofluorane entre CAM (concentración alveolar mínima) 1-1.5, estable a nivel cardiovascular durante el periodo transanestésico, con tiempos quirúrgico de $60 \mathrm{~min}$ y anestésico de 80 min, emersión de plano anestésico por lisis metabólica, sin reversión farmacológica, ni monitoreo de la relajación muscular. Con presencia de reflejos protectores de vía área se realiza extubación con aparición a los pocos segundos de polipnea, taquipnea, uso de músculos accesorios, por lo que se inicia manejo con ventilación asistida. Hay evidencia de 
aumento de la presión inspiratoria sin conseguir respuesta clínica adecuada, se administra aminofilina $250 \mathrm{mg} \mathrm{IV}$, seguida de 2 disparos de salbutamol inhalado, presentando expectoración con secreción asalmonada y estertores generalizados a la auscultación, hipoxemia severa con $\mathrm{SpO}_{2}$
$50 \%$ y $\mathrm{FiO}_{2} 100 \%$, por lo cual se decide realizar inducción anestésica con instrumentación de la vía aérea y se traslada a la unidad de cuidados intensivos. Gasometría arterial $\mathrm{pH}$ 7.23, $\mathrm{pCO}_{2}$ 55, $\mathrm{pO}_{2} 86$, Lac 1.9, $\mathrm{HCO}_{3} 23, \mathrm{BE}-4.6, \mathrm{FiO}_{2}$, $100 \%$, PAFI 86. Se ordenó radiografía de tórax (figura 3).

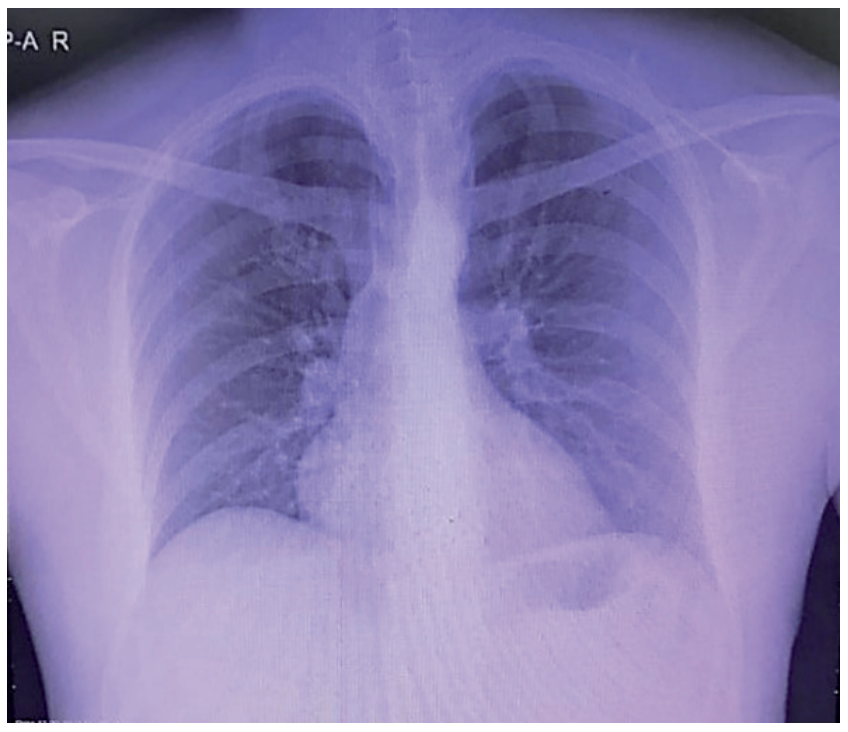

Figura 1. Radiografía de tórax posteroanterior al momento del ingreso. Tejidos blandos sin alteraciones, silueta cardiomediastínica conservada, ángulos costofrénicos y costodiafragmáticos libres, sin lesiones pleuro pulmonares. Fuente: los autores.

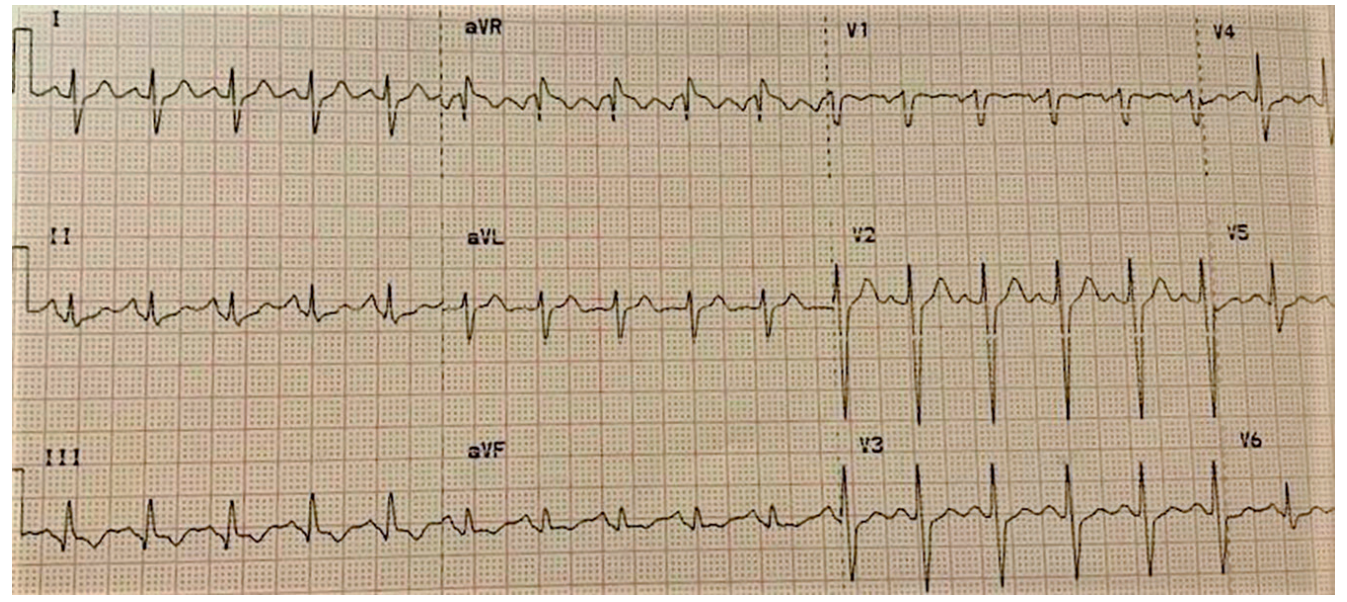

Figura 2. Electrocardiograma de ingreso, ritmo sinusal, frecuencia cardiaca 110 latidos por minuto, intervalo PR conservado, complejo QRS angosto, punto J isoeléctrico, segmento QT corto, adecuada progresión de la onda R. Sin trastornos de la repolarización. Fuente los autores. 


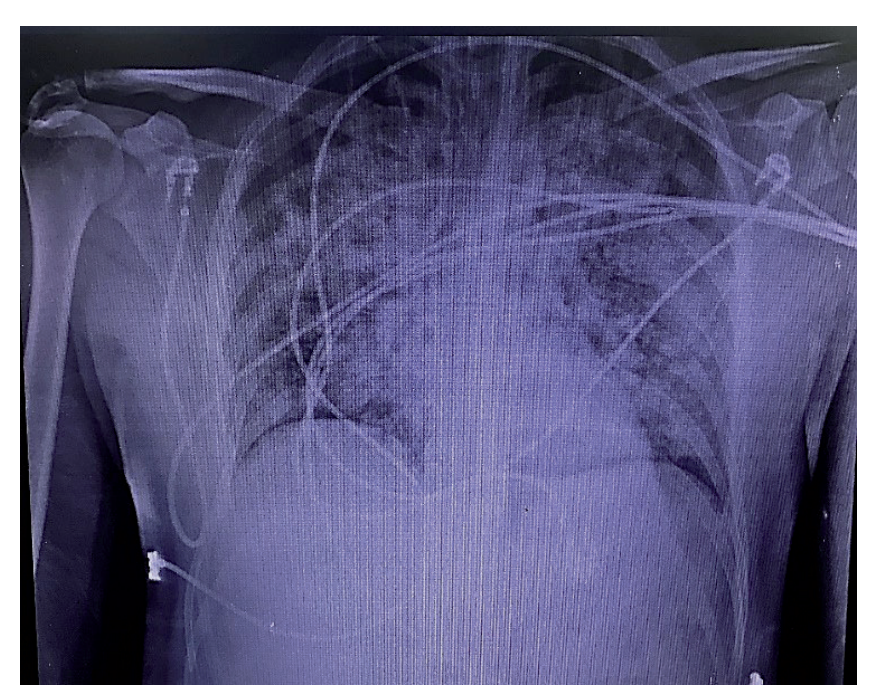

Figura 3. Radiografía de tórax anteroposterior al ingreso a terapia intensiva. Silueta mediastínica respetada. Ángulos costofrénicos y costodiafragmáticos libres, con imagen de infiltrados alveolares en parches diseminados en ambos campos pulmonares. Fuente: los autores.

Durante su estancia en la unidad de cuidados intensivos se mantuvo con ventilación mecánica dentro de parámetros de protección pulmonar controlado por volumen. Se tomó electrocardiograma el cual resultó sin cambios dinámicos y biomarcadores cardiacos no significativos que descartaron componente cardiogénico. CK 143 U/L (30-223), CK MB 7 UI/L (0.0-10.0) troponina I negativa. Se mantuvo con apoyo diurético tipo furosemida para disminución de líquido intersticial pulmonar, con estancia en la unidad de cuidados intensivos de 3 días en ventilación mecánica protectora. Se avanzó con manejo ventilatorio logrando extubación satisfactoria; control radiográfico (figura 4).

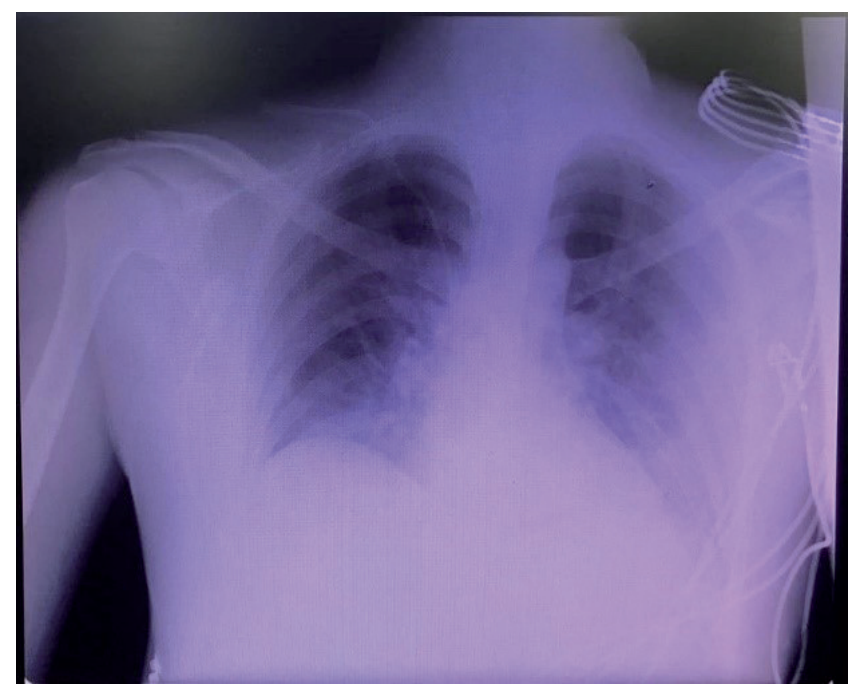

Figura 4. Radiografía de tórax AP al tercer día de estancia en terapia intensiva, sin lesiones de tejidos blandos, silueta cardiomediastínica conservada, ángulos costo frénicos y costodiafragmáticos libres, sin lesiones pleuropulmonares. Fuente: los autores.
El paciente evolucionó a la mejoría clínica y paraclínica con resolución satisfactoria del padecimiento. Egresó a la sala general y después se dio de alta.

\section{DISCUSIÓN}

El edema pulmonar agudo posextubación también conocido como edema pulmonar por presión negativa, es una clase no cardiogénica, que se divide en 2 tipos. El I se produce por un aumento de la presión inspiratoria contra una obstrucción proximal de la vía aérea, en el caso clínico presentado debido a un espasmo laríngeo, generando aumento en la presión transcapilar que conlleva a una mayor permeabilidad y a extravasación de líquido al espacio alveolar ${ }^{3}$, evidenciado por cambios clínicos, infiltrados alveolares radiográficos y esputo asalmonado.

La ocupación alveolar interrumpe el intercambio gaseoso facilitando una rápida hipoxemia e hipercapnia, lo cual causa vasoconstricción a nivel pulmonar mediada por el influjo de catecolaminas, en especial epinefrina, aumentando la probabilidad de fuga transcapilar. Lo anterior se explica por la ley de Starling ${ }^{4}$, con aumento de la presión capilar que supera la oncótica capilar, llevando a un edema transudado. En una respiración normal se genera una presión negativa de alrededor -5 a $-7 \mathrm{~cm} \mathrm{H}_{2} \mathrm{O}$, que en el contexto de una obstrucción aguda superior puede incrementarse hasta-100 $\mathrm{cm} \mathrm{H}_{2} \mathrm{O}$. Este aumento en la presión se transmite por continuidad a la pared ventricular incrementando el estrés y la postcarga según ley de Laplace ${ }^{5}$, que podría afectar el gasto cardiaco. Además se cree que el bloqueo neuromuscular residual puede jugar un papel preponderante en la fisiopatología de esta entidad, ya que los músculos laríngeos tardan más en recuperarse que el diafragma. ${ }^{5}$ En nuestro caso no se contaba con monitoreo de la relajación neuromuscular, situación por demás común en la práctica de la anestesiología sobre todo en pacientes que se creen sin factores de riesgo y llevados a procedimientos quirúrgicos de baja complejidad, pero además debido a ausencia del equipo necesario para monitoreo de la relajación.

Según la literatura encontrada, el edema agudo pulmonar posextubación tiene mayor incidencia entre pacientes ASA I y ASA II, con predominio del sexo masculino, posiblemente debido a que tienen un aparato respiratorio con mayor capacidad de respuesta ante una noxa. En un estudio observacional realizado por Tsai, Pei-Hsin y col. durante un tiempo de 8.5 años, el cual incluyó un total de 85.561 pacientes quienes recibieron anestesia general, su incidencia fue de $0.094 \%$, por otro lado, otros autores reportan hasta $11 \%$.

Dependiendo de la severidad de la hipoxemia y la clínica de la obstrucción se puede requerir manejo ventilatorio asistido con presión positiva hasta reintubación como en nuestro caso $^{5}$, de manera que se convierte en un tema de interés para muchas especialidades médicas. El 
tratamiento de esta entidad incluye minimizar los factores de riesgo asociados, tales como uso del tubo endotraqueal o dispositivos supraglóticos, secreciones que obstruyan la vía aérea, lesión directa por la laringoscopia repetida, uso de ventilación mecánica y monitoreo de la relajación muscular como uno de los principales detonantes del edema agudo pulmonar posextubación. Puede requerir tratamiento y vigilancia en unidad de cuidados intensivos, oxigeno suplementario, soporte ventilatorio con medidas de protección pulmonar, así como uso de un diurético que permita disminuir la precarga y dar soporte a la reabsorción de líquido intersticial. ${ }^{5}$ Como se reporta en la literatura el periodo de resolución puede durar entre 24 y 72 horas ${ }^{6}$, debido a que se suprime el factor desencadenante de la hipoxemia asociado con un mecanismo de reabsorción hídrica capilar indemne. ${ }^{7}$

\section{CONCLUSIONES}

El edema pulmonar agudo posextubación es una forma de presentación rara que lleva a una falla ventilatoria hipoxémica que puede compartir similitudes clínicas con el síndrome de dificultad respiratoria del adulto, cursa con baja incidencia por lo regular subvalorada y puede poner en riesgo potencial la vida del paciente, por ende debe tenerse en cuenta a la hora de llevar a pacientes ASA I y ASA II a procedimientos que requieran anestesia general, de manera que es fundamental como se ve en varios de los reportes a nivel mundial ${ }^{8}$, contar con un monitoreo de la relajación muscular a la hora de realizar la emersión y extubación, ya que esto minimizará en gran medida el factor de riesgo que supone una relajación muscular residual. En general es considerada una entidad benigna que mejora con tratamiento adecuado dentro de un periodo de 48 horas en promedio. $^{9}$

\section{CONFLICTO DE INTERESES}

Ninguno de los autores recibió alguna remuneración o ayuda financiera para desarrollar el trabajo. No existe ningún conflicto de interés por parte de alguno de los autores.

\section{REFERENCIAS}

1. Tsai PH, Wang JH, Huang SC, Lin YK, Lam CF. Characterizing postextubation negative pressure pulmonary edema in the operating room - a retrospective matched case-control study. Perioper Med (Lond). 2018;7:28. doi: 10.1186/s13741-018-0107-6.

2. More GP, Durve RS. Acute Pulmonary Oedema: A Post- Operative Complication Due to Neostigmine and Post Obstructive Pulmonary Oedema in a Case of Tonsillectomy. J Clin Diagn Res. 2015;9(6):UD05-6. doi: 10.7860/JCDR/2015/13072.6067

3. Rodrigues Silva LA, Guedes AA, Salgado Filho MF, Miranda Chaves LF, Paiva Araújo, F. Negative pressure pulmonary edema: report of four cases and review of the literature. Rev Bras Anestesiol. 2019;69(2):222-226. doi: 10.1016/j.bjan.2018.11.005

4. Gogia AR, Bajaj J, Sahni A, Saigal D. Negative-pressure pulmonary oedema in a patient undergoing shoulder arthroscopy. Indian J Anaesth. 2012;56(1):62-5. doi: 10.4103/0019-5049.93347

5. Bhattacharya M, Kallet R, Ware L, Matthay M. Negative Pressure Pulmonary Edema. Chest. 2016;150(4):927-933. doi: 10.1016/j. chest.2016.03.043

6. Chang Wei. Negative-pressure pulmonary edema after laparoscopic appendectomy. JAAPA. 2019;32(8):28-30. doi: 10.1097/01. JAA.0000574524.09174.bb

7. Hao, David \& Basnet, Sijan \& Melnick, Stephen \& Kim, James. (2019). Negative pressure pulmonary edema-related diffuse alveolar hemorrhage associated with Sevoflurane and cigarette smoking. J Community Hosp Intern Med Perspect. 2019;9(3):247251. doi: 10.1080/20009666.2019.1608140

8. Liu R, Wang J, Zhao G, Su, Z. Negative pressure pulmonary edema after general anesthesia: A case report and literature review. Medicine (Baltimore). 2019;98(17):e15389. doi: 10.1097/ MD.0000000000015389

9. Xiong, Jun \& Sun, Yongxing. (2019). Negative pressure pulmonary edema: a case report. BMC Anesthesiology. 2019;19(1):63. doi: 10.1186/s12871-019-0730-x. 